Li Y., F.Li, A. Emrouznejad, L. Liang, Q. Xie (2018) Allocating the Fixed Cost: An Approach based on Data Envelopment Analysis and Cooperative Game, Annals of Operations Research, Accepted,

https://doi.org/10.1007/s10479-018-2860-9

\title{
Allocating the Fixed Cost: An Approach based on Data Envelopment Analysis and Cooperative Game
}

\author{
Yongjun $\mathrm{Li}^{\mathrm{a}}$ \\ E-mail: lionli@ustc.edu.cn \\ Feng Li ${ }^{\mathrm{a}, *}$ \\ E-mail: lfeng90@mail.ustc.edu.cn \\ Ali Emrouznejad ${ }^{b}$ \\ E-mail: a.emrouznejad@aston.ac.uk \\ Liang Liang ${ }^{\mathrm{a}, \mathrm{c}}$ \\ E-mail: 1liang@ustc.edu.cn \\ Qiwei Xie \\ Email: qiwei.xie@ia.ac.cn
}

\footnotetext{
${ }^{\text {a }}$ School of Management, University of Science and Technology of China, Hefei, Anhui Province, PR China

${ }^{\mathrm{b}}$ Aston Business School, Aston University, Birmingham, UK

${ }^{c}$ School of Management, Hefei University of Technology, Hefei, Anhui Province, PR China

${ }^{\mathrm{d}}$ CAS Center for Excellence in Brain Science and Intelligence Technology, Beijing, PR China
}

* Feng $\mathrm{Li}$ is the corresponding author. 


\title{
Allocating the fixed cost: An approach based on data envelopment analysis and cooperative game
}

\author{
Yongjun Li, Feng Li, Ali Emrouznejad, Liang Liang, Qiwei Xie
}

\begin{abstract}
Allocating the fixed cost among a set of users in a fair way is an important issue both in management and economic research. Recently, Du et al. (2014) proposed a novel approach for allocating the fixed cost based on the game cross-efficiency method by taking the game relations among users in efficiency evaluation. This paper proves that the novel approach of $\mathrm{Du}$ et al. (2014) is equivalent to the efficiency maximization approach of $\mathrm{Li}$ et al. (2013), and may exist multiple optimal cost allocation plans. Taking into account the game relations in the allocation process, this paper proposes a cooperative game approach, and uses the nucleolus as a solution to the proposed cooperative game. The proposed approach in this paper is illustrated with a dataset from the prior literature and a real dataset of a steel and iron enterprise in China.
\end{abstract}

Keywords: Data Envelopment Analysis (DEA), Fixed cost allocation, Cooperative game, Nucleolus 


\section{Introduction}

The fixed cost, which refers to the expense of building a common platform by an organization for its subunits ( $\mathrm{Li}$ et al., 2009), frequently appears in real applications such as the advertisement expenditure of a manufacturer across its retailers (Cook and Kress, 1999), the transportation cost within a global supply chain (Vidal and Goetschalckx, 2001), and the cost of a common communication cable among its users (Beasley, 2003). A natural question arises about how to allocate the fixed cost of a common platform among its various users in an equitable manner.

Recently, data envelopment analysis (DEA), which is a classical non-parametric mathematical programming approach for relative efficiency evaluation (An et al., 2018; Li et al., 2018a, 2018ff; Wu et al., 2018), becomes a novel approach in solving the fixed cost allocation problem for it has several advantages (Si et al., 2013). First, DEA can successfully address multiple attributes (inputs and outputs), and the fixed cost allocation problem always refers to multiple attribute decision making problems. Second, the common platform's users (called as decision making units or "DMUs" in DEA) are independent, homogeneous, and comparable, which satisfies the requirement of homogeneity of DMUs in applying DEA to approach the fixed cost allocation problem (Dyson et al., 2001; Li et al., 2009). Third, DEA provides decision makers (DMs) the possibility to consider the effect of feasible allocation plans on performance evaluation (Li et al., 2009).

DEA-based fixed cost allocation approaches in literature can be divided into three categories. The first is based on the principle of efficiency-invariance, which assumes that the post-allocation efficiency of each DMU should be the same as its pre-allocation efficiency. Cook and Kress (1999) first propose this principle in allocating the fixed cost and develop several linear programs to obtain an equitable allocation. In order to obtain a unique cost allocation, some additional weight constraints such as the cone ratios (Charnes et al., 1989, 1990) may be added to these linear programs. Later, the approach of Cook and Kress (1999) is extended by Jahanshahloo et al. (2004), Cook and Zhu (2005), Lin (2011a, b) and Mostafaee 
(2013). Recently, Lin et al. (2016) proposed a new proportional sharing method for allocating the fixed cost based on two assumptions of efficiency invariance and zero slack simultaneously. Jahanshahloo et al. (2017) and Li et al. (2017) determined a unique allocation plan based on common weights and efficiency invariance principles simultaneously. Lin and Chen (2016) extended the efficiency invariance principle to super-efficiency situations, and then suggested three models, which guarantee piratical feasibility, to allocating a fixed cost as well as allocating a fixed resource along with sharing a common output target. However, Beasley (2003) and Li et al. (2013) find that the cost allocation based on the efficiency invariance principle may be determined entirely by the input side of DMUs, no matter how much difference among their outputs.

The second is the efficiency-maximization approach, which allocates the fixed cost based on the principle of maximizing the sum or average of post-allocation efficiencies of all DMUs. Beasley (2003) first applies such a novel principle but needs solving several non-linear models, and later is developed by Amirteimoori and Kordrostami (2005), Fang and Zhang (2008), Li et al. (2009), Amirteimoori and Tabar (2010), Khodabakhshi and Aryavash (2014) and Li et al. (2018d). Li et al. (2013) prove that non-linear models of Beasley (2003) can be changed to be linear since there exists a common set of weights that makes all DMUs (weakly) efficient, and the cost allocations based on the efficiency-maximization principle can be represented by a set of equations. Si et al. (2013) prove that the set of equations is the same as the traditional proportional sharing approach in one-dimensional case, and therefore call the set of equations as the extended proportional sharing approach. Most recently, Lin and Chen (2017) studied a situation where the allocated cost is a complement to the original inputs. Their approach considered the production level from a size point of view and is always feasible under both variable returns to scale and constant returns to scale properties. Yu et al. (2016), Zhu et al. (2017) and Li et al. (2018b) extended the fixed cost allocation problem to network situations by considering the internal two-stage processes, all three methods are implemented under the efficiency-maximization assumption. Li et al. (2018e) considered the feasibility of 
possible allocation plans and suggested a new non-egoistic principle, which states that each DMU should propose its allocation proposal in such a way that the maximal cost would be allocated to itself. Further, the optimal allocation plan is generated in a way that maximizes the efficiency scores for all DMUs.

The last is the game-based approach which takes into account the game relation among DMUs. This game relation can be understood since the less cost one DMU afford, the more the others. Nakabayashi and Tone (2006) first address the game relation among DMUs and use the cooperative game to solve the "egoist's dilemma" in the fixed cost allocation. Their approach directly defines the allocated cost as a ratio between the weighted inputs (outputs) and the weighted overall inputs (outputs), and doesn't take into account the fixed cost in efficiency evaluation of DMUs. Du et al. (2014) creatively apply the game cross-efficiency approach to addressing the game relation in the efficiency evaluation. The numerical example in Du et al. (2014) shows there may exist multiple cost allocations and these cost allocations make all DMUs (weakly) efficient, which is similar to the extended proportional sharing approach in Li et al. (2013). Li et al. (2018d) propose a DEA-game cross-efficiency approach for allocating the fixed cost, where all DMUs focus more on the cross-efficiency improvements than the allocated costs.

These observations motivate us to address two questions as follows:

1. What is the relation between the game cross-efficiency approach in Du et al. (2014) and the extended proportional sharing approach in Li et al. (2013)?

2. How to take into account the game relation among DMUs in allocating the fixed cost when there exist multiple cost allocations?

This paper first addresses the relation between the two approaches and finds that the game cross-efficiency method of Du et al. (2014) is equivalent to the extended proportional sharing method of Li et al. (2013). Then, all the feasible cost allocations can be represented as the extended proportional sharing equations. Based on the set of equations, this paper takes into account the game relation among DMUs, and proposes a cooperative game approach to allocate the fixed cost.

The remainder of this paper is organized as follows. Section 2 introduces the novel 
game cross-efficiency method of Du et al. (2014) and the extended proportional sharing method of Li et al. (2013), and then address the relations between the two approaches. Section 3 proposes a cooperative game DEA approach to obtain a unique fixed cost allocation, and applies the nucleolus as a solution to the cooperative game. Section 4 illustrates the proposed approach with two numerical examples, one from previous literature and the other a real application from a company in China. Conclusions and future research are given in the last section.

\section{Preliminaries}

This section first introduces the classical CCR model (Charnes et al., 1978), the game cross-efficiency method from Du et al. (2014) and the extended proportional sharing method from Li et al. (2013) and then addresses the relation between the two methods.

\subsection{Efficiency measurement taking into account the fixed cost}

Suppose there are $n$ homogenous DMUs, and each DMU consumes $m$ inputs to produce $s$ outputs. Denote the input vector and output vector of $D M U_{j}(j=1, \ldots, n)$ as $X_{j}=\left(x_{1 j}, \ldots, x_{m j}\right)$ and $Y_{j}=\left(y_{1 j}, \ldots, y_{s j}\right)$, respectively. The CCR efficiency of $D M U_{d}(d=1, . ., n)$ can be calculated as follows:

$$
\begin{aligned}
& \theta_{d}^{*}=\operatorname{Max} \frac{\sum_{r=1}^{s} u_{r} y_{r d}}{\sum_{i=1}^{m} v_{i} x_{i d}} \\
& \text { s.t. } \frac{\sum_{r=1}^{s} u_{r} y_{r j}}{\sum_{i=1}^{m} v_{i} x_{i j}} \leq 1, \quad \forall j \\
& u_{r}, v_{i}, \geq 0, \quad \forall r, i .
\end{aligned}
$$

Here $u_{r}, v_{i}$ are unknown weights attached to the $r^{\text {th }}$ output and the $i^{\text {th }}$ input, respectively, and the optimal objective function $\theta_{d}^{*}$ is defined as the CCR efficiency score of $D M U_{d}$.

Suppose the total fixed cost $R$ should be covered by $n$ DMUs, and $D M U_{j}(j=1, \ldots, n)$ affords a non-negative cost $R_{j}$ such that 


$$
\sum_{j=1}^{n} R_{j}=R, R_{j} \geq 0, \forall j .
$$

Formula (2) ensures that the sum of allocated fixed costs precisely equals to $R$, and the amount of allocated cost $R_{j}$ to each $D M U_{j}(j=1, \ldots, n)$ ranges from zero to $R$.

By taking into account the allocated fixed cost, the post-allocation efficiency of $D M U_{d}(d=1, \ldots, n)$ can be calculated as follows:

$$
\begin{aligned}
E_{d}^{*}= & \operatorname{Max} \frac{\sum_{r=1}^{s} u_{r} y_{r d}}{\sum_{i=1}^{m} v_{i} x_{i d}+v_{m+1} R_{d}} \\
\text { s.t. } & \frac{\sum_{r=1}^{s} u_{r} y_{r j}}{\sum_{i=1}^{m} v_{i} x_{i j}+v_{m+1} R_{j}} \leq 1, \quad \forall j \\
& \sum_{j=1}^{n} R_{j}=R \\
& R_{j} \geq 0, \quad \forall j \\
& u_{r}, v_{i} \geq 0, v_{m+1}>0, \quad \forall r, i .
\end{aligned}
$$

It is noteworthy that the allocated cost in model (3) is treated as an extra input and attached with a positive weight $\left(v_{m+1}>0\right)$. For details on this treatment, readers can refer to Beasley (2003).

Based on the literature review, the first cost allocation approach based on the principle of efficiency invariance finds cost allocations which make $\theta_{d}^{*}=E_{\mathrm{d}}{ }^{*}$. The other two cost allocation approaches are introduced as follows.

\subsection{The game cross-efficiency method}

Based on the game cross-efficiency concept, Du et al. (2014) adjust model (3) as follows:

$$
\begin{array}{ll}
E_{d}^{*}(d)=\operatorname{Max} \frac{\sum_{r=1}^{s} u_{r}^{d} y_{r d}}{\sum_{i=1}^{m} v_{i}^{d} x_{i d}+v_{m+1}^{d} R_{d}^{d}} \\
\text { s.t. } & e_{j} \leq \frac{\sum_{r=1}^{s} u_{r}^{d} y_{r j}}{\sum_{i=1}^{m} v_{i}^{d} x_{i j}+v_{m+1}^{d} R_{j}^{d}} \leq 1, \quad \forall j \\
& \sum_{j=1}^{n} R_{j}^{d}=R \\
& R_{j}^{d} \geq 0, \quad \forall j \quad \\
& u_{r}^{d}, v_{i}^{d}, v_{m+1}^{d} \geq \varepsilon, \quad \forall r, i,
\end{array}
$$

The major difference between model (3) and (4) is that model (4) adds a lower 
bound $e_{j}$ on the efficiency of $\mathrm{DMU}_{\mathrm{j}}$. This adjustment can ensure that $D M U_{d}$ maximizes its own efficiency score in choosing a cost allocation without reducing other DMUs' efficiency scores. Du et al. (2014) initially set $e_{j}=\theta_{j}{ }^{*}(j=1,2, \ldots, \mathrm{n})$, where $\theta_{j}{ }^{*}$ is the CCR efficiency score of $\mathrm{DMU}_{\mathrm{j}}$ based on model (1). Here, $\varepsilon$ is a sufficiently small positive value and $\varepsilon=10^{-6}$ in the numerical example of Du et al. (2014).

Model (4) can be transformed to be a linear programming by the Cooper-Charnes transformation such that $1 /\left(\sum_{i=1}^{m} v_{i}^{d} x_{i d}+v_{m+1}^{d} R_{d}^{d}\right)=\tau, \mu_{r}^{d}=\tau u_{r}^{d}, w_{i}^{d}=\tau v_{i}^{d}, w_{m+1}^{d}=\tau v_{m+1}^{d}$, and $r_{j}^{d}=w_{m+1}^{d} R_{j}^{d}$ as follows:

$$
\begin{array}{ll}
E_{d}^{*}(d)=\operatorname{Max} \quad \sum_{r=1}^{s} \mu_{r}^{d} y_{r d} \\
\text { s.t. } & \sum_{r=1}^{s} \mu_{r}^{d} y_{r j}-\sum_{i=1}^{m} w_{i}^{d} x_{i j}-r_{j}^{d} \leq 0, \quad \forall j \\
& e_{j} \sum_{i=1}^{m} w_{i}^{d} x_{i j}+e_{j} r_{j}^{d}-\sum_{r=1}^{s} \mu_{r}^{d} y_{r j} \leq 0, \quad \forall j \\
& \sum_{i=1}^{m} w_{i}^{d} x_{i d}+r_{d}^{d}=1 \\
& \sum_{j=1}^{n} r_{j}^{d}=w_{m+1}^{d} R \\
& r_{j}^{d} \geq 0, \quad \forall j \\
& \mu_{r}^{d}, w_{i}^{d}, w_{m+1}^{d} \geq \varepsilon, \quad \forall r, i .
\end{array}
$$

Denoting the optimal solution to model (5) as $\mu_{r}^{d^{*}}(\forall r), w_{i}^{d^{*}}(\forall i), w_{m+1}^{d^{*}}$, and $r_{j}^{d^{*}}(\forall j)$, the $d$-cross-efficiency for each $D M U_{j}(j=1, \ldots, n)$ (Doyle and Green, 1994) can be calculated by

$$
e_{j}(d)=\frac{\sum_{r=1}^{s} \mu_{r}^{d^{*}} y_{r j}}{\sum_{i=1}^{m} w_{i}^{d^{*}} x_{i j}+r_{j}^{d^{*}}}, \quad \forall j
$$

Then the cross-efficiency for $\operatorname{DMU}_{j}(j=1, \ldots, n)$ is most commonly calculated as the arithmetic mean value of all of its $d$-cross efficiencies (Doyle and Green, 1994; Li et al., 2018c) as follows:

$$
e_{j}=\frac{1}{n} \sum_{d=1}^{n} e_{j}(d)=\frac{1}{n} \sum_{d=1}^{n} \frac{\sum_{r=1}^{s} \mu_{r}^{d^{*}} y_{r j}}{\sum_{i=1}^{m} w_{i}^{d^{*}} x_{i j}+r_{j}^{d^{*}}}, \quad \forall j .
$$

Hence, the algorithm of cross-efficiency iterative method proposed by $\mathrm{Du}$ et al. (2014) can be summarized as below: 
Step 1: Solve model (1) and obtain $\theta_{j}^{*}(j=1, \ldots, n)$. Let $e_{j}=e_{j}^{1}=\theta_{j}^{*}$.

Step 2: Solve model (5) for each $D M U_{d}(d=1, \ldots, n)$.

Let $e_{j}^{t+1}=\frac{1}{n} \sum_{d=1}^{n} \frac{\sum_{r=1}^{s} \mu_{r}^{d^{*}}\left(e_{j}^{t}\right) y_{r j}}{\sum_{i=1}^{m} w_{i}^{d^{*}}\left(e_{j}^{t}\right) x_{i j}+r_{j}^{d^{*}}\left(e_{j}^{t}\right)}, \forall j, \quad$ where $\mu_{r}^{d^{*}}\left(e_{j}^{t}\right), \quad w_{i}^{d^{*}}\left(e_{j}^{t}\right), \quad$ and $r_{j}^{d^{*}}\left(e_{j}^{t}\right)$ are optimal values of $\mu_{r}^{d}, w_{i}^{d}$, and $r_{j}^{d}$, respectively, when $e_{j}=e_{j}^{t}$.

Step 3: If $\left|e_{j}^{t+1}-e_{j}^{t}\right|<\varepsilon, \forall j$, where $\varepsilon$ is a pre-specified small enough positive value ( $\varepsilon=10^{-6}$ in Du et al. (2014)), then the iterative algorithm terminates. If not, let $e_{j}=e_{j}^{t+1}$ and go to step 2 again.

When the iterative algorithm terminates, each $D M U_{d}(d=1, \ldots, n)$ gives an allocation plan $R_{j}^{d^{*}}=\frac{r_{j}^{d^{*}}\left(e_{j}^{t+1}\right)}{w_{m+1}^{d^{*}}\left(e_{j}^{t+1}\right)}(j=1, \ldots, n)$, and the average of the above allocations $R_{j}=\frac{1}{n} \sum_{d=1}^{n} R_{j}^{d^{*}}, \forall j$, is taken as the final fixed cost allocated to $D M U_{j}(j=1, \ldots, n)$. In addition, the numerical example in $\mathrm{Du}$ et al. (2014) shows the optimal cross-efficiency for each $D M U_{j}$ equals one and there may exist multiple cost allocations.

\subsection{The extended proportional sharing method}

Li et al. (2013) use model (3) to maximize the efficiency of each DMU, and prove that there exist some cost allocations which make each DMU's efficiency be one, even based on a common set of weights across all DMUs such that

$$
\begin{aligned}
& \frac{\sum_{r=1}^{s} u_{r} y_{r j}}{\sum_{i=1}^{m} v_{i} x_{i j}+v_{m+1} R_{j}}=1, \quad \forall j \\
& \sum_{j=1}^{n} R_{j}=R \\
& R_{j} \geq 0, \quad \forall j \\
& u_{r}, v_{i} \geq 0, v_{m+1}>0, \quad \forall r, i .
\end{aligned}
$$

By inserting $r_{j}=v_{m+1} R_{j}$, system (8) can be changed to be the following form: 
$r_{j}=\sum_{r=1}^{s} u_{r} y_{r j}-\sum_{i=1}^{m} v_{i} x_{i j}, \quad \forall j$

$\sum_{j=1}^{n} r_{j}=v_{m+1} R$

$r_{j} \geq 0, \quad \forall j$

$u_{r}, v_{i} \geq 0, v_{m+1}>0, \quad \forall r, i$.

The fixed cost allocated to $D M U_{j}(j=1, \ldots, n)$ can be denoted as $R_{j}=r_{j} / v_{m+1}$. Li et al. (2013) prove that cost allocations based on system (9) can be generated by a common set of weights across all DMUs. Si et al. (2013) find that the system (9) in the one-dimensional case equals to the standard proportional sharing method, and therefore call it as the extended proportional sharing method.

\subsection{Relationship of these two methods}

\subsubsection{Equivalence}

This subsection addresses the relations between the game cross-efficiency method of Du et al. (2014) and the extended proportional sharing method of Li et al. (2013). Here, we introduce two theorems.

Theorem 1. Each fixed cost allocation under a common set of weights based on system (9) can satisfy the algorithm of the game cross-efficiency method.

Proof: See Appendix 1.

This theorem shows that there exist efficient fixed cost allocation plans under a common set of weights based on system (9) that are also optimal solutions to the cross-efficiency iterative procedure. This result is consistent with the connotation of common weights, indicating that equal valuations of the input-output measures in the reference set (i.e., common weights) can be eventually accepted by all bargainers. Further, as Li et al. (2013) indicated that there will be multiple possible allocation plans that can satisfy the constraints of system (9), thus there will be also multiple allocation plans derived from the cross-efficiency iterative procedure.

Theorem 2. When the algorithm of the game cross-efficiency method terminates, the resulted fixed cost allocation can be generated based on system (9) under a common set of weights.

Proof: See Appendix 2.

Theorem 2 shows that any fixed cost allocation resulted from the cross-efficiency iterative procedure can be realized based on system (9) using a set of common 
weights. Although DMUs will not necessarily select identical relative weights in the cross-efficiency iterative procedure, a common set of weights can be used to replace the negotiation results yet without changing the allocation plan derived from the cross-efficiency iterative procedure. Hence, using a set of common weights automatically realizes the game cross-efficiency equilibrium in the fixed cost problem.

Based on Theorem 1 and 2, a corollary is obtained as follows:

Corollary 1. The optimal cost allocation of the game cross-efficiency method is equivalent to that of the extended proportional sharing method under a common set of weights based on system (9).

Proof: See Appendix 3.

Corollary 1 shows the equivalence of the two cost allocaiton methods, one is the game cross-efficiency method of Du et al. (2014), and the other is the extended proportional sharing method of Li et al. (2013). This interesting finding shows (a) the efficiency maximization cost allocation approach in fact considers the game relation among DMU in efficiency evaluation; and (b) all cost allocations based on the method of Du et al. (2014) can be represented as the syetem (9).

However, Li et al. (2013) show there may exist multiple cost allocations based on system (9). Readers can refer to the Appendix 4 for a detail discussion. In this case, DMUs still have flexibility of bargainning (game) in allocating the fixed cost. Then, the second question arises that how to take into account the game relation among DMUs in allocating the fixed cost when there exist multiple cost allocations.

\section{A cooperative game DEA approach}

This section proposes a cooperative game DEA approach to solve the second question. The cooperation and competition relations among DMUs exist at the same time. For example, DMUs are cooperative in jointly using a common platform rather than set up the platform independently, since this cooperative relation can save individual DMUs' costs. And also individual DMUs are competitive to cover the fixed cost. Therefore, to address such cooperation and competition relations among DMUs, a cooperative game is proposed in this section. 
Based on the framework of Nakabayashi and Tone (2006) and Lozano (2012), we make following assumptions to develop the cooperative game among all DMUs:

(1) DMUs are generally selfish. This principle implies that each DMU wants to minimize its allocated cost (or maximize its profit from our perspective);

(2) DMUs would like to participate in the grand coalition, and arrive at an equitable and acceptable allocation plan $z=\left(z_{1}, \ldots, z_{n}\right)$. We will demonstrate this point using the characteristic function as explained below.

\subsection{Characteristic function}

Let a coalition $S$ be a subset of the player set $N=\{1, \ldots, n\}$, and its inputs and outputs of coalition $S$ are denoted as $x_{i}(S)=\sum_{d \in S} x_{i d}$ and $y_{r}(S)=\sum_{d \in S} y_{r d}$. We propose model (10) to calculate the upper bound of fixed cost allocated to coalition $S$.

$$
\begin{array}{ll}
C(S)=\operatorname{Max} \sum_{r=1}^{s}\left(u_{r} / v_{m+1}\right) \sum_{d \in S} y_{r d}-\sum_{i=1}^{m}\left(v_{i} / v_{m+1}\right) \sum_{d \in S} x_{i d} \\
\text { s.t. } & r_{j}=\sum_{r=1}^{s} u_{r} y_{r j}-\sum_{i=1}^{m} v_{i} x_{i j}, \quad \forall j \\
& \sum_{j=1}^{n} r_{j}=v_{m+1} R \\
& r_{j} \geq 0, \quad \forall j \\
& u_{r}, v_{i} \geq 0, v_{m+1}>0, \quad \forall r, i .
\end{array}
$$

We can convert model (10) into a linear programming by setting $\mu_{r}=u_{r} / v_{m+1}, v_{i}=w_{i} / v_{m+1}$, and $R_{j}=r_{j} / v_{m+1}$.

$$
\begin{array}{ll}
C(S)=\operatorname{Max} \sum_{r=1}^{s} \mu_{r} \sum_{d \in S} y_{r d}-\sum_{i=1}^{m} w_{i} \sum_{d \in S} x_{i d} \\
\text { s.t. } & R_{j}=\sum_{r=1}^{s} \mu_{r} y_{r j}-\sum_{i=1}^{m} w_{i} x_{i j}, \quad \forall j \\
& \sum_{j=1}^{n} R_{j}=R \\
& R_{j} \geq 0, \quad \forall j \\
& \mu_{r}, w_{i} \geq 0, \quad \forall r, i .
\end{array}
$$

For any coalition $S \subseteq N$, the optimal objective function value $C(S)$ of model (11) can be regarded as the maximal "psychological price", since each coalition (or each DMU) is selfish to cover the cost share as low as possible. Therefore, the cost share of each coalition (DMU) should not be more than the optimal objective function of model (11). Here, when the coalition $S$ just has one DMU, we write $C(\{j\})$ as $C(j)$ for 
short.

Based on model (11), we define a characteristic function for the coalition $S$ as follows:

Definition 1: For any subset $S \subset N$, its characteristic function is defined as $V(S)=\sum_{j \in S} C(j)-C(S)$

Note $\sum_{j \in S} C(j)-C(S)$ denotes the gap between the sum of maximal "psychological prices" for individual DMU in $S$ and the corresponding price of the coalition $S$. The more the savings of the cost share of the coalition $S$, the bigger the value of $V(S)$. Therefore, $V(S)$ can be defined as the profit (gain) by forming the coalition $S$. All coalitions aim to maximize their profits.

The characteristic function $V(S)$ has following properties.

Proposition 2. $V(\varnothing)=0, V(N)=\sum_{j \in N} C(j)-R$.

Proof. See Appendix 5.

Theorem 3. The characteristic function $V(S)$ satisfies the super-additivity such that $V(S)+V(T) \leq V(S \cup T), \quad S, T \subseteq N$ and $S \cap T=\varnothing$.

Proof. See Appendix 6.

Theorem 3 shows the game $(N, V)$ forms a cooperative game, which have nonempty cores (Shapley, 1967) and give stable fixed cost allocations (Lozano, 2012). In addition, the game $(N, V)$ is a balanced game as follows:

Theorem 4. The cooperative game $(N, V)$ is a balanced game.

Proof. See Appendix 7.

Thus, the game $(N, V)$ belongs to transferable utility cooperative games. Based on the Definition 1, the transferable utility $V(S)$ is the "profit" (or money). The total payoff (or profits) of $V(S)$ can be distributed to its members (DMUs) based on several famous solutions to the cooperative game. Solutions can be the kernel, core, nucleolus, stable set, bargaining set, Shapley value, and so on (Shapley, 1967; Kruś and Bronisz, 2000; Nakabayashi and Tone, 2006; Lozano, 2012). For details, readers can refer to Owen (2013).

\subsection{Nucleolus-based allocation}


This section takes the nucleolus solution as an example to show how to obtain a fixed cost allocation, and other solution-based game allocations can be gotten in a similar way. Theoretically, the nucleolus-based game solution always exists and is unique (Schmeidler, 1969), which is an important feature for the studied fixed cost allocation problem. Suppose the nucleolus-based cost allocation of the cooperative game DEA $(N, V)$ be $z=\left(z_{1}, \ldots, z_{n}\right)$, it should satisfy the following rationalities:

1) Individual rationality: $C(j)-z_{j} \geq V(j)=0, \forall j \in N$.

2) Coalition rationality: $\sum_{j \in S} C(j)-\sum_{j \in S} z_{j} \geq V(S), \forall S \subset N$.

3) Collective rationality: $\sum_{j \in N} C(j)-\sum_{j \in N} z_{j}=V(N)=\sum_{j \in N} C(j)-R$.

The individual rationality ensures that the generated allocation brings improvement in the received profit for each individual DMU. The coalition rationality ensures that there exists no coalition that has incentives to quit the grand coalition. Last, the collective rationality ensures that all profits are distributed (i.e., the total fixed costs are actually allocated).

Based on the nucleolus solution, we need to give a definition to measure the effect of the cost allocation $z=\left(z_{1}, \ldots, z_{n}\right)$ on the happiness of the coalition $S$ as follows:

Definition 2: Let $z=\left(z_{1}, \ldots, z_{n}\right)$ be an imputation for the cooperative game DEA $(N, V)$, and its excess value of coalition $S$ is denoted as $e(S, z)=\left(\sum_{j \in S} C(j)-\sum_{j \in S} z_{j}\right)-V(S)$.

Based on Definition 1, $e(S, z)=C(S)-\sum_{j \in S} z_{j}$, and thus the excess value measures the distance from the cost allocation $z=\left(z_{1}, \ldots, z_{n}\right)$ to the worst result $C(S)$, and reflects the "degree of happiness" of coalition $S$ to the allocation $z$. The larger the excess value, the higher the "degree of happiness". If we sort the excess values of all coalitions $S \subseteq N, S \neq \varnothing$ in descending order, a vector $\theta(z)$ can be defined as follows: 


$$
\theta(z)=\left(\theta_{1}(z), \ldots, \theta_{2^{n}-2}(z)\right)=\left(e\left(S_{1}, z\right), \ldots, e\left(S_{2^{n}-2}, z\right)\right)
$$

where $e\left(S_{1}, z\right) \geq e\left(S_{2}, z\right) \geq \ldots \geq e\left(S_{2^{n}-2}, z\right)$.

Denote the set of all feasible cost allocations to the cooperative game DEA $(N, V)$ as $\mathrm{Z}$, then the nucleolus is the set of feasible distributions that maximizes $\theta(z)$ by the lexicographic order (Schmeidler, 1969) as follows:

$$
\psi(z)=\{z \in Z \mid \theta(z) \geq \theta(y), \forall y \in Z\} .
$$

\subsection{Computation algorithm}

To calculate the nucleolus-based allocation, the following max-min model is proposed based on the framework of Maschler et al. (1979).

$$
\begin{aligned}
& \underset{\mu, w}{\operatorname{Max}} \min _{S} e(S, z) \\
& \text { s.t. } z_{j}=\sum_{r=1}^{s} \mu_{r} y_{r j}-\sum_{i=1}^{m} w_{i} x_{i j}, \quad \forall j \\
& \sum_{j=1}^{n} z_{j}=R \\
& z_{j} \geq 0, \quad \forall j \\
& \mu_{r}, w_{i} \geq 0, \quad \forall r, i .
\end{aligned}
$$

Let $\beta=\min _{S} e(S, z)$, then the above program can be transformed into a linear one:

$$
\begin{aligned}
\underset{\mu, w}{\operatorname{Max}} \beta & \\
\text { s.t. } & z_{j}=\sum_{r=1}^{s} \mu_{r} y_{r j}-\sum_{i=1}^{m} w_{i} x_{i j}, \quad \forall j \\
& \sum_{j=1}^{n} z_{j}=R \\
& \sum_{j \in S} C(j)-\sum_{j \in S} z_{j}-V(S) \geq \beta, \quad \forall S \subseteq N, S \neq \varnothing \\
& z_{j} \geq 0, \quad \forall j \\
& \mu_{r}, w_{i} \geq 0, \quad \forall r, i .
\end{aligned}
$$

If we denote the optimal solution to model (14) as $\left(\beta_{1}^{*}, z_{j}^{*}, \forall j\right)$, then all coalitions can be divided into two subsets,

$$
\begin{aligned}
& \Gamma_{1}=\left\{S \mid \sum_{j \in S} C(j)-\sum_{j \in S} z_{j}-V(S)=\beta_{1}^{*}, \forall S \subseteq N, S \neq \varnothing\right\}, \\
& \Gamma_{2}=\left\{S \mid \sum_{j \in S} C(j)-\sum_{j \in S} z_{j}-V(S)>\beta_{1}^{*}, \forall S \subseteq N, S \neq \varnothing\right\} .
\end{aligned}
$$

Next, we maximize the smallest excess value for $\Gamma_{2}$. This process is repeated until the largest excess values of all coalitions are determined. The final optimal imputation 
(the final fixed cost allocation) is uniquely given by $\left(z_{j}^{*}, \forall j \in N\right)$. The computation algorithm can be summarized as follows:

Step 1: Let $l=1$. Denote the optimal solution to model (14) as $\left(\beta_{1}^{*}, \mu_{1 r}^{*}, w_{1 i}^{*}, z_{1 j}^{*}, \forall r, i, j\right)$. Then the corresponding excess value of each coalition can be calculated via definition 2. If $e(S, z)=\beta_{1}^{*}$, then denote the coalition set with the same excess value $\beta_{1}^{*}$ as $\Gamma_{1}=\left\{S \mid \sum_{j \in S} C(j)-\sum_{j \in S} z_{j}-V(S)=\beta_{1}^{*}, \forall S \subseteq N, S \neq \varnothing\right\}$. From this we get $\sum_{j \in S} z_{j}=\sum_{j \in S} C(j)-V(S)-\beta_{1}^{*}$, and we let $n_{1}$ denote the rank of the input-output matrix $\left(\sum_{j \in S} Y_{j}, \sum_{j \in S} X_{j}\right), S \in \Gamma_{1}$ of coalitions with the same excess value $\beta_{1}^{*}$. Then the other coalitions form a set denoted by $\Gamma_{2}=\left\{S \mid \sum_{j \in S} C(j)-\sum_{j \in S} z_{j}-V(S)>\beta_{1}^{*}, \forall S \subseteq N, S \neq \varnothing\right\}$. Obviously, we have $\Gamma_{0}=\Gamma_{1} \cup \Gamma_{2}$ and $\Gamma_{0}=\left\{S_{1}, \ldots, S_{2^{n}-2}\right\}$.

Step 2: If $n_{1}=m+s$, then the algorithm terminates and the optimal solution $\left(\beta_{1}^{*}, \mu_{1 r}^{*}, w_{1 i}^{*}, z_{1 j}^{*}, \forall r, i, j\right)$ is unique (the reason can be seen in Appendix 8). On the other hand, if $n_{1}<m+s$, then go to step 3 .

Step 3: Let $l=l+1$ and solve the following general model:

$$
\begin{array}{lll}
\beta_{l}^{*}= & \operatorname{Max}_{\mu, w} \beta \\
\text { s.t. } & \sum_{j=1}^{n} z_{j}=R \\
& z_{j}=\sum_{r=1}^{s} \mu_{r} y_{r j}-\sum_{i=1}^{m} w_{i} x_{i j}, \quad \forall j & \\
& \sum_{j \in S} C(j)-\sum_{j \in S} z_{j}-V(S)=\beta_{1}^{*}, \quad \forall S \in \Gamma_{1} \\
& \sum_{j \in S} C(j)-\sum_{j \in S} z_{j}-V(S)=\beta_{2}^{*}, \quad \forall S \in \Gamma_{3} \\
& \vdots \\
& \sum_{j \in S} C(j)-\sum_{j \in S} z_{j}-V(S)=\beta_{l-1}^{*}, \quad \forall S \in \Gamma_{2 l-3} \\
& \sum_{j \in S} C(j)-\sum_{j \in S} z_{j}-V(S) \geq \beta, \quad \forall S \in \Gamma_{2 l-2} \\
& z_{j} \geq 0, \quad \forall j & \\
& \mu_{r}, w_{i} \geq 0, \quad \forall r, i .
\end{array}
$$

We get the optimal solution $\left(\beta_{l}^{*}, \mu_{l r}^{*}, w_{l i}^{*}, z_{l j}^{*}, \forall r, i, j\right)$ from model (17) and the set of 
$\Gamma_{2 l-2}$ can be also divided into two subsets:

$$
\begin{aligned}
& \Gamma_{2 l-1}=\left\{S \mid \sum_{j \in S} C(j)-\sum_{j \in S} z_{j}-V(S)=\beta_{l}^{*}, \forall S \subseteq N, S \neq \varnothing\right\}, \\
& \Gamma_{2 l}=\left\{S \mid \sum_{j \in S} C(j)-\sum_{j \in S} z_{j}-V(S)>\beta_{l}^{*}, \forall S \subseteq N, S \neq \varnothing\right\} .
\end{aligned}
$$

Then the rank of the input-output matrix $\left(\sum_{j \in S} Y_{j}, \sum_{j \in S} X_{j}\right), S \in \Gamma_{1} \cup \Gamma_{3} \cup \ldots \cup \Gamma_{2 l-1}$ of coalitions with deterministic excess values from $\beta_{1}^{*}$ to $\beta_{l}^{*}$ is denoted as $n_{l}$.

Step 4: If $n_{l}=m+s$, the algorithm terminates, and the optimal solution $\left(\beta_{l}^{*}, \mu_{l r}^{*}, w_{l i}^{*}, z_{l j}^{*}, \forall r, i, j\right)$ is also unique. If $n_{l}<m+s$, then go to step 3 again.

As discussed above, the nucleolus-based allocation plan maximizes the "degree of happiness" for coalitions of DMUs by the lexicographic order. More importantly, the nucleolus-based allocation plan first addresses the least happy coalition, aiming to maximize its "degree of happiness" on the fixed cost allocation results. Intuitively, favoring the least coalition is a generous philosophy and will cause less resistance in implementing the allocation plan in organizations. In such a way the acceptability of the resulted allocation plan will be improved, which will favor the decision maker's effort in implementing the resulted allocation plan. By repeatedly maximizing the "degree of happiness" across all coalitions of DMUs, the decision maker will face the least difficulty and resistance to allocate the total fixed cost, and as a result it would be easier to completely allocate the total fixed cost of a common platform among its various users.

\section{Illustrative applications}

This section uses two datasets to illustrate the proposed cooperative game DEA approach, one is a dataset from Cook and Kress (1999) and the other is a real data of a steel and iron enterprise from China in 2015.

\subsection{A numerical example}

Table 1 shows the dataset from Cook and Kress (1999), and it has 12 DMUs and each DMU consumes 3 inputs to generate 2 outputs. The total fixed cost $R=100$ is to be allocated. Based on model (11), the maximal allocated cost each DMU can afford is 
calculated and shown in the second column of Table 2.

Table 1 A dataset from Cook and Kress (1999).

\begin{tabular}{cccccc}
\hline DMU & Input 1 & Input 2 & Input 3 & Output 1 & Output 2 \\
\hline $\mathbf{1}$ & 350 & 39 & 9 & 67 & 751 \\
$\mathbf{2}$ & 298 & 26 & 8 & 73 & 611 \\
$\mathbf{3}$ & 422 & 31 & 7 & 75 & 584 \\
$\mathbf{4}$ & 281 & 16 & 9 & 70 & 665 \\
$\mathbf{5}$ & 301 & 16 & 6 & 75 & 445 \\
$\mathbf{6}$ & 360 & 29 & 17 & 83 & 1070 \\
$\mathbf{7}$ & 540 & 18 & 10 & 72 & 457 \\
$\mathbf{8}$ & 276 & 33 & 5 & 78 & 590 \\
$\mathbf{9}$ & 323 & 25 & 5 & 75 & 1074 \\
$\mathbf{1 0}$ & 444 & 64 & 6 & 74 & 1072 \\
$\mathbf{1 1}$ & 323 & 25 & 5 & 25 & 350 \\
$\mathbf{1 2}$ & 444 & 64 & 6 & 104 & 1199 \\
\hline
\end{tabular}

Based on model (14), the optimal excess value is $\beta_{1}^{*}=1.326$, and its corresponding coalition set $\Gamma_{1}=\{\{1,7,8,9,11\},\{5,7,9,11,12\},\{3,4,6,7,8,10,12\},\{2,3,4,6,7,10,12\},\{N-\{11\}\}\}$, such that $\beta_{S}^{*}=1.326, \forall S \in \Gamma_{1}$. The computation algorithm terminates, since $\operatorname{rank}(M)=5=m+s$, where $M=\left(\sum_{j \in S} Y_{j}, \sum_{j \in S} X_{j}\right), \forall S \in \Gamma_{1}$. Therefore, the fixed cost allocation can be determined as shown in the third column of Table 2, which is the nucleolus-based solution to the cooperative game $(N, V)$. It is clear that the nucleolus-based solution shows a fairness concern by sympathizing and assisting vulnerable groups (i.e., the group has less excess value), thus the resulted allocation plan is of fairness. The calculation process of nucleolus-based allocation plan consistently sympathizes and assists vulnerable groups, thus the resulted allocation plan would be more acceptable and easily implemented in organizations in real managerial applications. In addition, the nucleolus-based allocation plan is very stable, and it is suitable for organizations consisting of a set of completing units.

For the convenience of comparison, cost allocations based on Cook and Kress (1999), Beasley (2003), Li et al. (2013), and Du et al. (2014) are also given in the last four columns of Table 2 . 
First, the second column shows that upper bound of the fixed cost each DMU can afford is accepted by all approaches, except Cook and Kress (1999). The reason is that Cook and Kress (1999) belongs to the efficiency-invariance approach, while the others belongs or equals to the efficiency-maximization approach. The cost allocation based on Cook and Kress (1999) shows that the cost allocation may be entirely due to the input side, not depend on their outputs (Beasley, 2003). Readers can refer to two pairs of DMUs (DMU9 and DMU11, DMU10 and DMU12). This finding does not exist for other approaches. For example, our approach shows that $\mathrm{DMU}_{10}$ and $\mathrm{DMU}_{11}$ are allocated less cost than DMU12 and $\mathrm{DMU}_{9}$, respectively.

Table 2 Fixed cost allocation results based on different approaches.

\begin{tabular}{ccccccc}
\hline DMU & $\begin{array}{c}\text { Upper } \\
\text { bound }\end{array}$ & $\begin{array}{c}\text { Our } \\
\text { approach }\end{array}$ & $\begin{array}{c}\text { Cook and } \\
\text { Kress (1999) }\end{array}$ & $\begin{array}{c}\text { Beasley } \\
(\mathbf{2 0 0 3})\end{array}$ & $\begin{array}{c}\text { Li et al. } \\
(\mathbf{2 0 1 3})\end{array}$ & $\begin{array}{c}\text { Du et al. } \\
(\mathbf{2 0 1 4})\end{array}$ \\
\hline $\mathbf{1}$ & 8.7859 & 7.27 & 14.52 & 6.78 & 6.38 & 5.79 \\
$\mathbf{2}$ & 9.6907 & 7.61 & 6.74 & 7.21 & 7.42 & 7.95 \\
$\mathbf{3}$ & 9.8192 & 6.57 & 9.32 & 6.83 & 6.68 & 6.54 \\
$\mathbf{4}$ & 12.7309 & 8.77 & 5.6 & 8.47 & 8.83 & 11.10 \\
$\mathbf{5}$ & 12.1649 & 6.99 & 5.79 & 7.08 & 7.63 & 8.69 \\
$\mathbf{6}$ & 19.1686 & 11.50 & 8.15 & 10.06 & 9.70 & 13.49 \\
$\mathbf{7}$ & 11.1340 & 4.63 & 8.86 & 5.09 & 4.28 & 7.10 \\
$\mathbf{8}$ & 12.9434 & 7.89 & 6.26 & 7.74 & 8.35 & 6.83 \\
$\mathbf{9}$ & 24.4768 & 14.05 & 7.31 & 15.11 & 15.87 & 16.68 \\
$\mathbf{1 0}$ & 17.2763 & 9.77 & 10.08 & 10.08 & 9.75 & 5.42 \\
$\mathbf{1 1}$ & 3.9468 & 1.33 & 7.31 & 1.58 & 0.46 & 0 \\
$\mathbf{1 2}$ & 22.5158 & 13.63 & 10.08 & 13.97 & 14.64 & 10.41 \\
\hline
\end{tabular}

Compared with these efficiency-maximization approaches in Beasley (2003), Li et al. (2013) and Du et al. (2014), our approach has an advantage in reducing the absolute amount difference between the maximal and minimal cost among these DMUs, since $12.72(=14.05-1.33)$ is less than $13.53,15.41$ and 16.68. As Li et al. (2009) suggested, a smaller gap among the allocated costs of DMUs will bring about less difficulty in implementing the allocation.

It is noteworthy that the cost allocation based on Du et al. (2014) is one of multiple optimal allocations. The proposed approach in this paper considers cooperation and competition relations among DMUs, and uses the cooperative game to allocate the fixed cost. From the perspective of the game, the resulted cost allocation in this paper 
may be more acceptable by DMUs as compared to other approaches.

Table 3 Difference of five methods on three conditions.

\begin{tabular}{lcccccc}
\hline \multicolumn{1}{c}{ Conditions } & \multicolumn{5}{c}{ Methods } \\
\cline { 2 - 6 } & $\mathrm{O}$ & $\mathrm{CK}$ & $\mathrm{B}$ & $\mathrm{L}$ & $\mathrm{D}$ \\
\hline Whether the method is linear? & YES & YES & NO & YES & YES \\
Whether the allocation is unique? & YES & YES & YES & YES & NO \\
Whether the method considers the game among & & & & & \\
DMUs. & & YES & NO & NO & NO & YES \\
\hline
\end{tabular}

In order to observe the difference of the above five methods clearly, we further use Table 3 to describe different conditions that these methods satisfied: (1) whether the method is linear? (2) Whether the allocation is unique? (3) Whether the method considers the game among DMUs. From the results, we find that both Cook and Kress (1999), Beasley (2003) and Li et al. (2013) can determine a unique allocation, but the game among DMUs is not addressed, and even a series of nonlinear programming is used in Beasley (2003), which makes its allocation more complex. Only our proposed approach satisfies these three conditions simultaneously.

\subsection{A real application in a steel and iron enterprise}

A steel and iron company in Anhui Province China has ten production lines. Governed by the environmental and energy regulations, the firm faces enormous pressure to improve the structure of production and to enhance the efficiency of energy utilization. In 2015, the firm spent 20 million Yuan (RMB) to upgrade and retrofit some essential production equipment. Therefore, it requires its production lines to cover the total cost. In this application, each production line is considered as an independent and homogeneous DMU, which has four inputs (Standard coal, Iron ore, New water, and Labor) and two outputs (Production of steel and iron) as shown in Table 4. To offset the upgrade cost completely, here the fixed cost to be allocated is supposed to be $R=2000$ (unit: ten thousand Yuan (RMB)).

Table 4 Input-output data of the steel and iron company.

\begin{tabular}{ccccccc}
\hline DMU & $\begin{array}{c}\text { Standard coal } \\
\text { (ton) }\end{array}$ & $\begin{array}{c}\text { Iron ore } \\
\text { (ton) }\end{array}$ & $\begin{array}{c}\text { New water } \\
\text { (ton) }\end{array}$ & $\begin{array}{c}\text { Labor } \\
\text { (person) }\end{array}$ & $\begin{array}{c}\text { Steel } \\
\text { (ton) }\end{array}$ & $\begin{array}{c}\text { Iron } \\
\text { (ton) }\end{array}$ \\
$\mathbf{1}$ & 74115 & 375944 & 2420 & 374 & 150236 & 41917 \\
$\mathbf{2}$ & 84713 & 381974 & 3464 & 224 & 106177 & 68738 \\
$\mathbf{3}$ & 76087 & 299056 & 2848 & 363 & 224356 & 42603 \\
\hline
\end{tabular}




\begin{tabular}{ccccccc}
\hline $\mathbf{4}$ & 109150 & 420650 & 1645 & 397 & 133291 & 48536 \\
$\mathbf{5}$ & 114462 & 356279 & 1661 & 324 & 301229 & 60390 \\
$\mathbf{6}$ & 104493 & 427276 & 3024 & 356 & 200101 & 60148 \\
$\mathbf{7}$ & 118825 & 392056 & 4220 & 351 & 253604 & 125641 \\
$\mathbf{8}$ & 159620 & 283154 & 3136 & 290 & 160096 & 102599 \\
$\mathbf{9}$ & 193873 & 391289 & 1976 & 341 & 160441 & 120832 \\
$\mathbf{1 0}$ & 146680 & 278386 & 2246 & 442 & 97847 & 49659 \\
\hline
\end{tabular}

Similar to Section 4.1, we first solve model (11) and get the upper bound of allocated costs for all coalitions of DMUs. Here we show the upper bound for individual DMU in the second column in Table 5. Further, we use model (14) to maximize minimum excess values (i.e., the degree of happiness) among all coalitions of DMUs. Within the first round, we get the optimal objective function $\beta_{1}^{*}=42.9418$ for two coalitions $\{1,3,6,8,9\}$ and $\{2,4,5,7,10\}$, and the corresponding allocation result and excess value for each individual DMU are given in the third and fourth columns of Table 5. Note that at this time it holds that $n_{1}=2<6$, thus we would turn into the second round. We directly solve model (17) and get the second minimum excess value $\beta_{2}^{*}=53.4210$ for four coalitions, that is, $\{4,6,8\}$, $\{3,6,9,10\},\{1,3,6,7,9,10\}$ and $\{1,2,3,4,5,6,7,8,9\}$. Now the equality $n_{2}=6=m+s$ holds, hence the algorithm terminates. As a result, the allocation derived from the second round was taken as the final nucleolus-based allocation of the upgrade costs, which is given in the last column of Table 5 .

Table 5 Fixed cost allocation results of the upgrade costs.

\begin{tabular}{ccccccc}
\hline DMU & $\begin{array}{c}\text { Upper } \\
\text { bound }\end{array}$ & Round 1 & $\begin{array}{c}\text { Excess } \\
\text { value }\end{array}$ & Round 2 & $\begin{array}{c}\text { Excess } \\
\text { value }\end{array}$ & Allocation \\
\hline $\mathbf{1}$ & 201.8171 & 89.6874 & 112.1298 & 87.1106 & 114.7064909 & 87.1106 \\
$\mathbf{2}$ & 262.3214 & 102.0070 & 160.3145 & 106.8298 & 155.4916676 & 106.8298 \\
$\mathbf{3}$ & 400.8508 & 161.1517 & 239.6990 & 154.3165 & 246.5342492 & 154.3165 \\
$\mathbf{4}$ & 170.7199 & 104.2614 & 66.4585 & 102.0796 & 68.64026791 & 102.0796 \\
$\mathbf{5}$ & 572.2772 & 320.6585 & 251.6187 & 314.1732 & 258.1039762 & 314.1732 \\
$\mathbf{6}$ & 261.0845 & 159.7399 & 101.3446 & 156.9450 & 104.139537 & 156.9450 \\
$\mathbf{7}$ & 626.1986 & 389.9909 & 236.2077 & 400.4142 & 225.7844092 & 400.4142 \\
$\mathbf{8}$ & 445.7973 & 269.6518 & 176.1455 & 273.6742 & 172.1231731 & 273.6742 \\
$\mathbf{9}$ & 641.9022 & 342.8513 & 299.0509 & 351.0359 & 290.8663374 & 351.0359 \\
$\mathbf{1 0}$ & 137.7383 & 60.0001 & 77.7382 & 53.4210 & 84.31727576 & 53.4210 \\
\hline
\end{tabular}


According to the final optimal fixed cost allocation results, four production lines (DMU5, $\mathrm{DMU}_{7}, \mathrm{DMU}_{8}$, and $\mathrm{DMU}_{9}$ ) would need to pay much more than other production lines, and contribute more than two-third of the total costs. This could be due to the fact that these four production lines have a higher upper bound on possible allocated costs. On the contrary, $\mathrm{DMU}_{1}, \mathrm{DMU}_{4}$ and $\mathrm{DMU}_{10}$ are characterized with the lowest upper bound, so they afford the least upgrade costs as compared to its peers. Additionally, if we turn our attention to the operation size for these ten production lines, we can find that all production lines have very similar ranks in both overall size and allocated cost in ascending order, and even four of these ten production lines $(3,4$, 7 and 9) have identical ranks in both overall size and allocated cost, as shown in Table 6. This fact implies that the allocation result derived from our proposed approach is implicitly consistent with the current input consumptions and output productions from a size point of view. That is to say, a DMU with larger scale is more likely to bear more cost, while less cost will be undertaken by DMUs with smaller sizes. This feature is an intuitive result and also demonstrates the validity of the proposed approach.

Table 6 Operation sizes and allocated costs.

\begin{tabular}{|c|c|c|c|c|c|c|c|c|c|c|}
\hline \multirow{2}{*}{$\frac{\mathrm{DMU}}{1}$} & \multicolumn{4}{|c|}{ Input size } & \multicolumn{2}{|c|}{ Output size } & \multirow{2}{*}{$\frac{\text { Overall size }}{0.0847}$} & \multirow{2}{*}{$\frac{\text { Rank }}{1}$} & \multirow{2}{*}{$\frac{\text { Allocated cost }}{87.1106}$} & \multirow{2}{*}{$\frac{\text { Rank }}{2}$} \\
\hline & 0.0627 & 0.1043 & 0.0908 & 0.1080 & 0.0841 & 0.0581 & & & & \\
\hline 2 & 0.0717 & 0.1059 & 0.1300 & 0.0647 & 0.0594 & 0.0953 & 0.0878 & 2 & 106.8298 & 4 \\
\hline 3 & 0.0644 & 0.0829 & 0.1069 & 0.1049 & 0.1255 & 0.0591 & 0.0906 & $\underline{5}$ & 154.3165 & $\underline{5}$ \\
\hline 4 & 0.0923 & 0.1167 & 0.0617 & 0.1147 & 0.0746 & 0.0673 & 0.0879 & $\underline{3}$ & 102.0796 & $\underline{3}$ \\
\hline 5 & 0.0968 & 0.0988 & 0.0623 & 0.0936 & 0.1685 & 0.0838 & 0.1006 & 6 & 314.1732 & 8 \\
\hline 6 & 0.0884 & 0.1185 & 0.1135 & 0.1028 & 0.1120 & 0.0834 & 0.1031 & 7 & 156.9450 & 6 \\
\hline 7 & 0.1005 & 0.1087 & 0.1584 & 0.1014 & 0.1419 & 0.1742 & 0.1309 & $\underline{10}$ & 400.4142 & $\underline{10}$ \\
\hline 8 & 0.1350 & 0.0785 & 0.1177 & 0.0838 & 0.0896 & 0.1423 & 0.1078 & 8 & 273.6742 & 7 \\
\hline 9 & 0.1640 & 0.1085 & 0.0742 & 0.0985 & 0.0898 & 0.1676 & 0.1171 & $\underline{9}$ & 351.0359 & $\underline{9}$ \\
\hline 10 & 0.1241 & 0.0772 & 0.0843 & 0.1277 & 0.0547 & 0.0689 & 0.0895 & 4 & 53.4210 & 1 \\
\hline
\end{tabular}

* The size of a particular measure is calculated as the ratio of the measure value to the aggregated value across all production lines. The overall size is an arithmetic mean value across all measure sizes in the same row.

\section{Conclusions}

It is notable that the cooperation and competition relations among DMUs 
simultaneously exist in allocating the fixed cost. This paper first finds that the novel game cross-efficiency approach of $\mathrm{Du}$ et al. (2014) is equivalent to the extended proportional sharing approach of Li et al. (2013). And both two approaches may give multiple cost allocations in the multi-dimensional case. This paper then considers the game relations in advance in the fixed cost allocation and proposes a cooperative game DEA approach. We define the super-additive characteristic function and adopts the nucleolus as a solution to the game. The resulted allocation scheme maximizes the "degree of happiness" of all coalitions through maximizing all excess values, which can be more acceptable to all DMUs. Finally, the proposed cooperative game DEA approach is illustrated with both a numerical example from previous literature and a real case of steel and iron enterprise from China.

Future research may introduce the fairness criterion, and propose an approach based on trade-off between the efficiency and fairness criterion. Besides, one can explicitly take the operation size into account, and the generated allocation plan is proportional to input usages and output productions from a size point of view.

\section{Acknowledgements}

The authors would like to thank the Editor of Annals of Operations Research and two anonymous reviewers for their kind work and valuable comments. We would also acknowledge helpful comments from Professor Joe Zhu. This research was financially

supported by the National Natural Science Foundation of China (Grant Nos. 71271196 and 71671172), the Youth Innovation Promotion Association of Chinese Academy of Sciences (CX2040160004), and Science Funds for Creative Research Groups of University of Science and Technology of China (No. WK2040160008). Professor Xie would like to thank the GreatWall Scholar Training Program of Beijing Municipality (CIT\&TCD 20180305) and Social Science Foundation of Beijing (16JDGLC005).

\section{References}

Amirteimoori, A., Kordrostami, S. (2005). Allocating fixed costs and target setting: a 
DEA-based approach. Applied Mathematics and Computation, 171(1), 136-151.

Amirteimoori, A., Tabar, M. M. (2010). Resource allocation and target setting in data envelopment analysis. Expert Systems with Applications, 37(4), 3036-3039.

An, Q., Meng, F., Ang, S., Chen, X. (2018). A new approach for fair efficiency decomposition in two-stage structure system. Operational Research, 18(1), 257-272.

Beasley, J.E. (2003). Allocating fixed costs and resources via data envelopment analysis. European Journal of Operational Research, 147(1), 198-216.

Charnes, A., Cooper, W.W., Rhodes, E. (1978). Measuring the efficiency of decision making units. European Journal of Operational Research, 2(6), 429-444.

Charnes, A., Cooper, W.W., Wei, Q.L., Huang, Z.M. (1989). Cone ratio data envelopment analysis and multi-objective programming. International Journal of Systems Science, 20(7), 1099-1118.

Charnes, A., Cooper, W.W., Huang, Z.M., Sun, D.B. (1990). Polyhedral cone-ratio DEA models with an illustrative application to large commercial banks. Journal of Econometrics, 46(1), 73-91.

Cook, W.D. Kress, M. (1999). Characterizing an equitable allocation of shared costs: A DEA approach. European Journal of Operational Research, 119(3), 652-661.

Cook, W.D., Zhu, J. (2005). Allocation of shared costs among decision making units: a DEA approach. Computers \& Operations Research, 32(8), 2171-2178.

Du, J., Cook, W. D., Liang, L., Zhu, J. (2014). Fixed cost and resource allocation based on DEA cross-efficiency. European Journal of Operational Research, 235(1), 206-214.

Doyle, J., Green, R. (1994). Efficiency and cross-efficiency in DEA: Derivations, meanings and uses. Journal of the Operational Research Society, 45(5), 567-578.

Dyson, R.G., Allen, R., Camanho, A.S., Podinovski, V.V., Sarrico, C.S., Shale, E.A. (2001). Pitfalls and protocols in DEA. European Journal of Operational Research, 132(2), 245-259.

Fang, L., Zhang, C.Q. (2008). Resource allocation based on the DEA model. Journal of the Operational Research Society, 59(8), 1136-1141.

Jahanshahloo, G.R., Lotfi, F.H., Shoja, N., Sanei, M. (2004). An alternative approach for equitable allocation of shared costs by using DEA. Applied Mathematics and Computation, 153(1), 267-274.

Kruś, L., Bronisz, P. (2000). Cooperative game solution concepts to a cost allocation problem. European Journal of Operational Research, 122(2), 258-271.

Khodabakhshi, M., Aryavash, K. (2014). The fair allocation of common fixed cost or revenue using DEA concept. Annals of Operations Research, 214(1), 187-194.

Jahanshahloo, G.R., Sadeghi, J., Khodabakhshi, M. (2017). Proposing a method for fixed cost allocation using DEA based on the efficiency invariance and common set of weights principles. Mathematical Methods of Operations Research, 85(2), 223-240. 
Li, F., Liang, L., Li, Y., Emrouznejad, A. (2018a). An alternative approach to decompose the potential gains from mergers. Journal of the Operational Research Society, doi.org/ 10.1080/01605682.2017.1409867.

Li, F., Song, J., Dolgui, A., Liang, L. (2017). Using common weights and efficiency invariance principles for resource allocation and target setting. International Journal of Production Research, 55(17), 4982-4997.

Li, F., Zhu, Q., Chen, Z. (2018b). Allocating a fixed cost across the decision making units with two-stage network structures. Omega, doi.org/10.1016/j.omega.2018.02.009.

Li, F., Zhu, Q., Chen, Z., Xue, H. (2018c). A balanced data envelopment analysis cross-efficiency evaluation approach. Expert Systems with Applications, 106, 154-168.

Li, F., Zhu, Q., Liang, L. (2018d). Allocating a fixed cost based on a DEA-game cross efficiency approach. Expert Systems with Applications, 96, 196-207.

Li, F., Zhu, Q., Liang, L. (2018e). A new data envelopment analysis based approach for fixed cost allocation. Annals of Operations Research, doi.org/10.1007/s10479-018-2819-x.

Li, F., Zhu, Q., Zhuang, J. (2018f). Analysis of fire protection efficiency in the United States: a two-stage DEA-based approach. OR Spectrum, 40(1), 23-68.

Li, Y., Yang, F., Liang, L., Hua, Z. (2009). Allocating the fixed cost as a complement of other cost inputs: A DEA approach. European Journal of Operational Research, 197(1), 389-401.

Li, Y., Yang, M., Chen, Y., Dai, Q., Liang, L. (2013). Allocating a fixed cost based on data envelopment analysis and satisfaction degree. Omega, 41(1), 55-60.

Lin, R. (2011a). Allocating fixed costs or resources and setting targets via data envelopment analysis. Applied Mathematics and Computation, 217(13), 6349-6358.

Lin, R. (2011b). Allocating fixed costs and common revenue via data envelopment analysis. Applied Mathematics and Computation, 218(7), 3680-3688.

Lin, R., Chen, Z. (2016). Fixed input allocation methods based on super CCR efficiency invariance and practical feasibility. Applied Mathematical Modelling, 40(9), 5377-5392.

Lin, R., Chen, Z. (2017). A DEA-based method of allocating the fixed cost as a complement to the original input. International Transactions in Operational Research, doi.org/10.1111/itor.12495.

Lin, R., Chen, Z., Li, Z. (2016). A new approach for allocating fixed costs among decision making units. Journal of Industrial and Management Optimization,12(1), 211-228.

Lozano, S. (2012). Information sharing in DEA: A cooperative game theory approach. European Journal of Operational Research, 222(3), 558-565.

Maschler, M., Peleg, B., Shapley, L.S. (1979). Geometric properties of the kernel, nucleolus, and related solution concepts. Mathematics of operations research, 4(4): 303-338.

Mostafaee, A. (2013). An equitable method for allocating fixed costs by using data envelopment analysis. Journal of the Operational Research Society, 64(3), 326-335. 
Nakabayashi, K., Tone, K. (2006). Egoist's dilemma: a DEA game. Omega, 34(2), 135-148.

Owen, G. (2013). Game theory (4 ${ }^{\text {th }}$ eds). Emerald Group Publishing Limited.

Si, X., Liang, L., Jia, G., Yang, L., Wu, H., Li, Y. (2013). Proportional sharing and DEA in allocating the fixed cost. Applied Mathematics and Computation, 219(11), 6580-6590.

Schmeidler, D. (1969). The nucleolus of a characteristic function game. SIAM Journal on Applied Mathematics, 17(6), 1163-1170.

Shapley, L.S. (1967). On balanced sets and cores. Naval Research Logistics Quarterly, 14(4), 453-460.

Vidal, C.J., Goetschalckx, M. (2001). A global supply chain model with transfer pricing and transportation cost allocation. European Journal of Operational Research, 129(1), 134-158.

Wu, J., Yu, Y., Zhu, Q., An, Q., Liang, L. (2018). Closest target for the orientation-free context-dependent DEA under variable returns to scale. Journal of the Operational Research Society. https://doi.org/10.1080/01605682.2017.1409865.

Yu, M.M., Chen, L.H., Hsiao, B. (2016). A fixed cost allocation based on the two-stage network data envelopment approach. Journal of Business Research, 69(5), 1817-1822.

Zhu, W., Zhang, Q., Wang, H. (2017). Fixed costs and shared resources allocation in two-stage network DEA. Annals of Operations Research. doi.10.1007/s10479-017-2599-8.

\section{Appendix}

\section{Appendix 1}

Theorem 1. Each fixed cost allocation under a common set of weights based on system (9) can satisfy the algorithm of the game cross-efficiency method.

Proof. The cost allocation under a common set of weights is presented as

$$
\begin{aligned}
& r_{j}=\sum_{r=1}^{s} u_{r} y_{r j}-\sum_{i=1}^{m} v_{i} x_{i j}, \quad \forall j \\
& \sum_{j=1}^{n} r_{j}=v_{m+1} R \\
& r_{j} \geq 0, \quad \forall j \\
& u_{r}, v_{i} \geq 0, v_{m+1}>0, \quad \forall r, i .
\end{aligned}
$$

Let $\hat{R}_{j}=\hat{r}_{j} / \hat{v}_{m+1}(j=1, \ldots, n)$ be an allocation associated with $\left(\hat{u}_{r}, \hat{v}_{i}, \hat{v}_{m+1}\right)$ in

(A1.1), and then $\left(\hat{u}_{r}, \hat{v}_{i}, \hat{v}_{m+1}, \hat{R}_{j}=\hat{r}_{j} / \hat{v}_{m+1}\right)$ is a feasible solution to model (4) (or linear model (5)), for it can satisfy all constraints of model (4), such that

$$
e_{j}(d)=\frac{\sum_{r=1}^{s} u_{r}^{d} y_{r j}}{\sum_{i=1}^{m} v_{i}^{d} x_{i j}+v_{m+1}^{d} R_{j}^{d}}=\frac{\sum_{r=1}^{s} \hat{u}_{r} y_{r j}}{\sum_{i=1}^{m} \hat{v}_{i} x_{i j}+\left(\sum_{r=1}^{s} \hat{u}_{r} y_{r j}-\sum_{i=1}^{m} \hat{v}_{i} x_{i j}\right)}=\frac{\sum_{r=1}^{s} \hat{u}_{r} y_{r j}}{\sum_{r=1}^{s} \hat{u}_{r} y_{r j}}=1, \quad \forall j .
$$


$\sum_{j=1}^{n} R_{j}=\sum_{j=1}^{n}\left(\hat{r}_{j} / \hat{v}_{m+1}\right)=\sum_{j=1}^{n} \hat{r}_{j} / \hat{v}_{m+1}=R$

Hence, we have also $e_{j}=\frac{1}{n} \sum_{d=1}^{n} e_{j}(d)=1, \forall j$ and $E_{d}^{*}(d)=1$. It means that $\left(\hat{u}_{r}, \hat{v}_{i}, \hat{v}_{m+1}, \hat{R}_{j}=\hat{r}_{j} / \hat{v}_{m+1}\right)$ is an optimal solution to model (4) and we cannot further improve the efficiency for any $D M U_{j}$. Then for any smaller enough positive $\varepsilon>0$, we have $\left|e_{j}^{t+1}-e_{j}^{t}\right|=0<\varepsilon$. The algorithm of cross-efficiency iterative method terminates.

Note that $\left(\hat{u}_{r}, \hat{v}_{i}, \hat{v}_{m+1}, \hat{R}_{j}=\hat{r}_{j} / \hat{v}_{m+1}\right)$ is chosen randomly based on (A1.1), so any fixed cost allocation under a common set of weights based on system (9) can satisfy the algorithm of the cross-efficiency iterative method.

\section{Appendix 2}

Theorem 2. When the algorithm of the game cross-efficiency method terminates, the resulted fixed cost allocation can be generated based on system (9) under a common set of weights.

Proof. It is proven by $\mathrm{Du}$ et al. (2014) that, when the cross-efficiency iterative algorithm terminates the optimal cross-efficiency for any $D M U_{j}$ equals one. Denote the optimal solution to the game cross-efficiency method as $\left(\hat{u}_{r}^{d^{*}}, \hat{v}_{i}^{d^{*}}, \hat{v}_{m+1}^{d^{*}}, \hat{r}_{j}^{d^{*}}\right)$. Based on formula (7) we have

$$
e_{j}=\frac{1}{n} \sum_{d=1}^{n} \frac{\sum_{r=1}^{s} \hat{u}_{r}^{d^{*}} y_{r j}}{\sum_{i=1}^{m} \hat{d}_{i}^{d^{*}} x_{i j}+\hat{r}_{j}^{d^{*}}}=1, \quad \forall j .
$$

Since the input-oriented $d$-cross-efficiency is no more than one, it must be that

$$
\frac{\sum_{r=1}^{s} \hat{u}_{r}^{d^{*}} y_{r j}}{\sum_{i=1}^{m} \hat{v}_{i}^{d^{*}} x_{i j}+\hat{r}_{j}^{d^{*}}}=1, \quad \forall d, j .
$$

Then, $\hat{r}_{j}^{d^{*}}=\sum_{r=1}^{s} \hat{u}_{r}^{d^{*}} y_{r j}-\sum_{i=1}^{m} \hat{v}_{i}^{d^{*}} x_{i j}, \quad \forall d, j$.

Further, we have

$$
\begin{aligned}
r_{j} & =\frac{1}{n} \sum_{d=1}^{n} r_{j}^{d^{*}}=\frac{1}{n} \sum_{d=1}^{n}\left(\sum_{r=1}^{s} \hat{u}_{r}^{d^{*}} y_{r j}-\sum_{i=1}^{m} \hat{v}_{i}^{d^{*}} x_{i j}\right) \\
& =\sum_{r=1}^{s}\left(\frac{1}{n} \sum_{d=1}^{n} \hat{u}_{r}^{d^{*}}\right) y_{r j}-\sum_{i=1}^{m}\left(\frac{1}{n} \sum_{d=1}^{n} \hat{v}_{i}^{d^{*}}\right) x_{i j}, \quad \forall j .
\end{aligned}
$$


Let $u_{r}=\frac{1}{n} \sum_{d=1}^{n} \hat{u}_{r}^{d^{*}}$ and $v_{i}=\frac{1}{n} \sum_{d=1}^{n} \hat{v}_{i}^{d^{*}}$, then we have system (A2.5).

$$
\begin{aligned}
& r_{j}=\sum_{r=1}^{s} u_{r} y_{r j}-\sum_{i=1}^{m} v_{i} x_{i j}, \quad \forall j . \\
& \sum_{j=1}^{n} r_{j}=\frac{1}{n} \sum_{j=1}^{n} \sum_{d=1}^{n} \hat{r}_{j}^{d^{*}}=\frac{1}{n} \sum_{d=1}^{n} \sum_{j=1}^{n} \hat{r}_{j}^{d^{*}}=\frac{1}{n} \sum_{d=1}^{n} v_{m+1} R=v_{m+1} R
\end{aligned}
$$

By combining system (A2.5) and the non-negative/positive constraints on variables, we get the same formulation as system (9). Therefore, when the cross-efficiency iterative algorithm terminates, the resulted final fixed cost allocation can be realized under a common set of weights based on system (9).

\section{Appendix 3}

Corollary 1. The optimal cost allocation of the game cross-efficiency method is equivalent to that of the extended proportional sharing method under a common set of weights based on system (9).

Proof. It can be easily proven by combining theorems 1 and 2 .

\section{Appendix 4}

Based on Corollary 1, all cost allocations based on Du et al. (2014) can be represented by system (9). And it can be transformed as follows:

$$
R=\left(\sum_{r=1}^{s} u_{r} \sum_{j=1}^{n} y_{r j}-\sum_{i=1}^{m} v_{i} \sum_{j=1}^{n} x_{i j}\right) / v_{m+1}, v_{m+1}>0 .
$$

In the one dimensional case, the allocation based on Formula (A4.1) is unique and the same as the standard proportional sharing method (Li et al., 2013; Si et al., 2013).

In the general multi-dimensional case, however, the two approaches of Du et al. (2014) and $\mathrm{Li}$ et al. (2013) may give multiple allocations, since there exist $(m+s+n+1)$ variables and $(n+1)$ equations in system (9). Based on Li et al. (2013) and Si et al. (2013), we present Proposition 1 here to show the non-uniqueness.

Proposition 1. According to the extended proportional sharing method based on system (9):

(i) The unique allocation can be obtained if the cost allocation problem is a one-dimensional case in which only one output measure is considered, i.e., $s=1$ and $m=0$;

(ii) Multiple allocations may be available if and only if $m+s>1$.

Proposition 1 can be easily proven using basic results in linear algebra, and here we omit the proof.

\section{Appendix 5}


Proposition 2. $V(\varnothing)=0, V(N)=\sum_{j \in S} C(j)-R$.

Proof. The first part is held automatically. For the second part,

$$
V(N)=\sum_{j \in N} C(j)-C(S)=\sum_{j \in N} C(j)-R \geq 0 .
$$

\section{Appendix 6}

Theorem 3. The characteristic function $V(S)$ satisfies the super-additivity property, i.e., we have $V(S)+V(T) \leq V(S \cup T)$, if $S, T \subseteq N$ and $S \cap T=\varnothing$.

Proof. $V(S)+V(T)=\sum_{j \in S} C(j)-C(S)+\sum_{j \in T} C(j)-C(T)$

$$
=\sum_{j \in S \cup T} C(j)-(C(S)+C(T))
$$

Based on the egoist's dilemma in Nakabayashi and Tone (2006), we can find that the fixed allocation problem in model (11) would be sub-additive. That is, $C(S \cup T) \leq C(S)+C(T)$ for any $S, T \subset N$. As a result, we have

$$
\begin{aligned}
V(S)+V(T) & =\sum_{j \in S \cup T} C(j)-(C(S)+C(T)) \\
& \leq \sum_{j \in S \cup T} C(j)-C(S \cup T) \\
& =V(S \cup T) \quad \forall S, T \subset N, S \cap T=\varnothing .
\end{aligned}
$$

\section{Appendix 7}

Theorem 4. The cooperative game $(N, V)$ is a balanced game.

Proof. Consider a vector $\lambda$ with $n^{2}-2$ nonnegative components $\lambda_{S}, S \subseteq N$, which satisfies that $\sum_{j \in S \subseteq N} \lambda_{S}=1, \forall j \in N$. Then, according to Shapley (1967) the game $(N, V)$ is said to be balanced if it holds $\sum_{S \subseteq N} \lambda_{S} V(S) \leq V(N)$.

According to model (11) and definition 1 on the characteristic function, we have 


$$
\begin{aligned}
& \sum_{S \subseteq N} \lambda_{S} V(S)=\sum_{S \subseteq N} \lambda_{S}\left[\sum_{j \in S} C(j)-C(S)\right] \\
& =\sum_{S \subseteq N} \lambda_{S}\left\{\sum_{j \in S} C(j)-\max _{\mu_{r}, w_{i}}\left[\sum_{r=1}^{s} \mu_{r}\left(\sum_{j \in S} y_{r j}\right)-\sum_{i=1}^{m} w_{i}\left(\sum_{j \in S} x_{i j}\right)\right]\right\} \\
& =\sum_{S \subseteq N} \lambda_{S}\left[\sum_{j \in S} C(j)\right]-\sum_{S \subseteq N} \lambda_{S}\left\{\max _{\mu_{r}, w_{i}}\left[\sum_{r=1}^{s} \mu_{r}\left(\sum_{j \in S} y_{r j}\right)-\sum_{i=1}^{m} w_{i}\left(\sum_{j \in S} x_{i j}\right)\right]\right\} \\
& \leq \sum_{S \subseteq N} \lambda_{S}\left[\sum_{j \in S} C(j)\right]-\max _{\mu_{r}, w_{i}} \sum_{S \subseteq N} \lambda_{S}\left[\sum_{r=1}^{s} \mu_{r}\left(\sum_{j \in S} y_{r j}\right)-\sum_{i=1}^{m} w_{i}\left(\sum_{j \in S} x_{i j}\right)\right] \\
& =\sum_{S \subseteq N} \lambda_{S}\left[\sum_{j \in S} C(j)\right]-\max _{\mu_{r}, w_{i}}\left\{\sum_{r=1}^{s} \mu_{r}\left[\sum_{S \subseteq N} \lambda_{S}\left(\sum_{j \in S} y_{r j}\right)\right]-\sum_{i=1}^{m} w_{i}\left[\sum_{S \subseteq N} \lambda_{S}\left(\sum_{j \in S} x_{i j}\right)\right]\right\} \\
& =\sum_{j \in N} C(j)\left(\sum_{j \in S \subseteq N} \lambda_{S}\right)-\max _{\mu_{r}, w_{i}}\left\{\sum_{r=1}^{s} \mu_{r}\left[\sum_{j \in N} y_{r j}\left(\sum_{j \in S \subseteq N} \lambda_{S}\right)\right]-\sum_{i=1}^{m} w_{i}\left[\sum_{j \in N} x_{i j}\left(\sum_{j \in S \subseteq N} \lambda_{S}\right)\right]\right\} \\
& =\sum_{j \in N} C(j)-\max _{\mu_{r}, w_{i}}\left[\sum_{r=1}^{s} \mu_{r}\left(\sum_{j \in N} y_{r j}\right)-\sum_{i=1}^{m} w_{i}\left(\sum_{j \in N} x_{i j}\right)\right]=\sum_{j \in N} C(j)-R=V(S) .
\end{aligned}
$$

The above inequality is an immediate result of Nakabayashi and Tone's (2006) egoist's dilemma.

Hence, the cooperative game $(N, V)$ is a balanced game.

\section{Appendix 8}

Combine equations in system (9) and equation $\sum_{j \in S} C(j)-\sum_{j \in S} z_{j}-V(S)=\beta_{1}^{*}$,

we have $C(S)-\beta_{1}^{*}=\sum_{r} \mu_{r} \sum_{j \in S} y_{r j}-\sum_{i} w_{i} \sum_{j \in S} x_{i j}, \forall S \in \Gamma_{1}$.

where $\mu_{r}=u_{r} / v_{m+1}, v_{i}=w_{i} / v_{m+1}$, and $R_{j}=r_{j} / v_{m+1}$. Apparently, it contains $m+s$

variables $\left(\mu_{r}, w_{i}, \forall r, i\right)$. If $n_{1}=m+s$, we have $m+s$ equations that are reciprocally linearly independent, then the unique solution can be obtained according to theories in Linear Algebra. Accordingly, the fixed cost allocation plan can be uniquely determined, and then the algorithm terminates. If $n_{1}<m+s$, the rank of coefficient matrix is smaller than the number of variables. As a result, there still leaves flexibility in the variables, and we cannot terminate the algorithm but go to step 3. A similar situation occurs in step 4. If $n_{l}=m+s$, then we get uniquely determined fixed cost allocation plan and terminate the algorithm, else do until there are $m+s$ linearly independent equations uniquely determining the variables and resulted allocation plan. 